\title{
25 Research Soure \\ Dendrobium officinale Orchid Extract could improve wound healing in diabetic mice
}

\section{Qiang-qiang Zhu ( $\nabla$ zhuqiang1125@163.com )}

Yunnan Agricultural University https://orcid.org/0000-0003-1109-5335

\section{Cai-jun Yu}

Yunnan Agricultural University

\section{Xiao-ying Yang}

Yunnan Agricultural University

\section{Qian-qian Pang}

Yunnan Agricultural University

Jian-fei Li

Yunnan Agricultural University

Ming-ming Li

Yunnan Agricultural University

\section{Xuan-jun Wang}

Yunnan Agricultural University

\section{Jun Sheng}

Yunnan Agricultural University

\section{Research}

Keywords: dendrobium officinale orchid extract, diabetes complication, p65 signaling pathway, wound healing

Posted Date: September 3rd, 2020

DOI: https://doi.org/10.21203/rs.3.rs-70025/v1

License: (9) This work is licensed under a Creative Commons Attribution 4.0 International License.

Read Full License 


\section{Abstract \\ Background}

In diabetes, delayed wound healing is one of the most prominent clinical manifestations and lacks satisfactory therapeutic schemes. Persistent inflammation impairs the healing process which occurs in the late phase of wound healing in diabetes mellitus (DM) mice. It has been report that dendrobium officinale (DE) could decrease inflammation and the NF-KB signaling pathway plays a critical role in regulated inflammation. The aim of the present study is to detect whether $D E$ attenuated inflammation by NF-KB to accelerate wound healing in diabetes.

\section{Methods}

Mice were induced to diabetes by streptozocin (STZ) for 15 days. Two circular 8-mm full-thickness wounds were created on the back and then received DE treatment for 18 days. The percentage of wound healing, re-epithelialization and collagen deposition were calculation to evaluation the effect of DE on wound healing. The expression levels of interleukin (IL)- $1 \beta$ and TNF- $\alpha$ in the wound site were determined by WB. Macrophage deposition and the type of macrophage were detected by WB and immunohistochemical staining. In addition, the expression of p65 was detected by WB.

\section{Results}

Herein, we observed that the late wound healing could be improved by dendrobium officinale orchid extract (DE) in streptozotocin (STZ)-induced DM mice. The accumulation of collagen was increased by DE treatment in the skin. We also found that the expression of pro-inflammation cytokines IL-1 $\beta$, and TNF-a were inhibited by DE treatment in the skin wounds of DM mice. Furthermore, the macrophages accumulation which increased in diabetes wound site, were inhibited by DE treatment in the DM mice wound skin. And the CD163 which is a marker of type 2 macrophage was increased by DE treatment. Moreover, we confirmed that DE could decrease the expression of iNos which is a marker of type 1 macrophage by $\mathrm{IHC}$ in the skin wounds. And the levels of p65 and p65 phosphorylation were decreased by $D E$.

\section{Conclusions}

Wound healing can be improved by DE through NF-KB signaling pathway to attenuated inflammation in STZ-induced diabetic mice.

\section{Background}


According to the WHO, diabetes affects 422 million people worldwide and the increase in prevalence is occurring at a faster rate in low - and middley - income countries ${ }^{1,2}$. Delay wound healing is one of the primary secondary complications of diabetes and presently lacks satisfactory treatment options ${ }^{2}$.

Diabetic patients exhibit delayed healing of acute wounds, which often develop into chronic ulcers in their feet and lower limbs ${ }^{1}$. Non-healing diabetic wounds represent a significant contributor to costs and poor quality of life. Non-healing wounds also leading cause of lower extremity amputation which leads to drastic increases in morbidity and mortality as the 5 -year mortality rate post amputation is over $50 \%{ }^{3}$. The management of diabetic wounds is focused primarily on debridement, offloading, antibiotic therapy, and in some cases, surgical revascularization including angioplasty and bypass ${ }^{4}$. And some new potential therapeutic intervention have been report to improved diabetes wounds, such as sustained delivery of growth factors, siRNA delivery, targeting micro RNA, and stem cell therapy ${ }^{5}$. However, even with these therapeutic managements, there are approximately accounts for $60 \%$ of non-traumatic amputations of the lower limb ${ }^{6}$, and nearly one-third of diabetic foot ulcers require surgery ${ }^{7}$. Now, the traditional therapies based on natural origin compounds, such as plant extracts, honey, and larvae, are interesting alternatives. These therapies offer new possibilities for the treatment of diabetes wound healing, enhancing the access to the healthcare, and allowing overcoming some limitations associated to the modern products and therapies, such as the high costs, the long manufacturing times, and the increase in the bacterial resistance ${ }^{8}$. Thus, traditional therapies agent could be propose to improve wound healing.

Wound healing is a complex biological process that involves a large number of cell types, growth factors, and cytokines which results in the restoration of tissue integrity ${ }^{9,10}$. Diabetes mellitus can cause impaired wound healing by affecting one or more biological mechanisms of the process ${ }^{10}$. Multiple and complex pathophysiological factors contribute to this failure to heal ${ }^{5}$. Diabetic wounds are characterized by delayed wound healing due to persistent inflammation ${ }^{11}$. The sustained inflammatory responses lead to inappropriate activation and tissue injury and even to increased susceptibility to invasive microorganisms and poor healing ${ }^{12}$.

Macrophages were exist in two different phenotypic states within the healing wound ${ }^{13}$, and the dysfunction of macrophage ${ }^{14}$ is a component of the pathogenesis of non-healing and poorly healing wounds ${ }^{15}$. So, macrophage is a determining factor in the low-grade inflammation associated with diabetes wound healing.

Natural compounds which therapeutic activities by anti-inflammatory, antimicrobial, and cell-stimulating properties have been used in skin wound care for many years ${ }^{8}$. Dendrobium candidum is a medicinal herb that has been employed in China and Southeast Asian countries ${ }^{16}$. The Dendrobium candidum display immunomodulatory, antioxidant, anticancer, and neuroprotective activities ${ }^{16}$. However, as a natural compound, whether it could be used in skin wound care is still unclear. Thus, we hypothesized 
that Dendrobium officinale orchid extract (DE) could improve diabetic wound healing by regulation the inflammation.

To prove our hypothesis, STZ-induced diabetes mice were used to study the effects of DE on wound healing. The results show that DE can be improved the late wound healing in DM mice. And inflammation was attenuated in the DE-treated DM mice. These findings suggest that DE can enhance wound healing, decrease the expression of p65, p65 phosphorylation, and decrease the pro-inflammation cytokines secretion. Hence, the identification effect of DE on wound healing by attenuate inflammation provides novel insights into therapeutic strategy for diabetic wounds and likely makes DE into a potentially novel medicine to treat chronic wounds.

\section{Methods}

\section{Animals}

Healthy virgin inbred ICR mice (7-8 weeks old, $31.2 \pm 2.8 \mathrm{~g}$, male) were purchased from CAVENS LAB ANIMAL Co., (Changzhou, China) and allowed to rest for 2 weeks. All mice were maintained in a controlled environment ( $12 \mathrm{~h}$ light/ $12 \mathrm{~h}$ dark cycle; relative humidity at $60 \%$; ambient temperature $24 \pm$ $1^{\circ} \mathrm{C}$ ) and were administered with standard laboratory food (ShooBree R Rat Food, Nanjing, China) and water ad libitum. All mice experiments were performed in the animal facility according to the institutional guidelines and were approved by the Institutional Animal Care and Use Committee of the Yunnan Agricultural University. Adverse events were not observed.

\section{Diabetes induction and monitoring}

To induce diabetes in mice, we administered a single injection of STZ (100 mg/ $\mathrm{kg}$ body weight, i.p.) (MilliporeSigma, Shanghai, China). STZ was freshly dissolved in cold citrate buffer $(0.05 \mathrm{M} ; \mathrm{pH} 4.5)$. Before STZ injection, all the mice were fasting overnight and weighed $(41.1 \pm 2.5 \mathrm{~g})$. The blood glucose levels (BGLs) of each mouse was measured using tail-vein blood samples by using a glucose meter (Sannuo, Changsha, China). The mice were housed in a controlled environment for two weeks before testing the BGLs. Mice with fasting BGLs $>17 \mathrm{mmol} / \mathrm{L}$ (about $306 \mathrm{mg} / \mathrm{dL}$ ) were confirmed to be diabetic and kept for up to 16 weeks to develop diabetic nephropathy as previously described ${ }^{17-19}$.

\section{Excisional wounding and treatment}

Mice were administered inhalational anesthesia using pentobarbital (30 mg/kg i.p.). Their dorsal fur was shaved and skin was cleaned with an alcohol swab. Two circular 8-mm full-thickness wounds were created on the back of each mouse with a dermal biopsy punch ${ }^{20}$. After the diabetic induced, mice were randomly divided into the following three groups ( $n=8$ per group): normal group, DM group, DE group. Normal and DM groups were treated with $1 \%$ carboxymethylcellulose (CMC) as placebo. For the DE treatment group, we administered as a single dose of $20 \mathrm{mg} / \mathrm{mL}$ DE dissolved in $1 \% \mathrm{CMC}$. The drug or placebo was topical applied on the wound. All groups were treated once daily and the wounds were not covered. 


\section{Assessment of wound healing}

As previously described, three blinded reviewers carried out independent verification of all wound healing assessment ${ }^{21}$. Mice were anesthetized and the wound surface area was recorded daily using a digital camera (5D marker $\Downarrow$, Canon, Japan). The straight edges were used as scales in photographs, and the wound area was calculated using NIH Image J Software (National Institute of Heath, Bethesda, MD, USA). The initial wound size was calculated immediately after wounding, and wound closure was assessed over time as the percentage of initial wound area.

\section{Histochemical staining}

Whole wounds were excised from the backside of mice using a 10-mm punch biopsy on post-wound day 8. Tissue samples of mice were fixed in $10 \%$ buffered formalin ( $0.1 \mathrm{M}$ phosphate buffer, $\mathrm{pH} 7.4$ ), embedded in paraffin wax (Solarbio, China), cut into 3- $\mu \mathrm{m}$ sections, and used for histopathological examination. The sections were randomly selected and stained with hematoxylin (Sigma, USA) and eosin (Solarbio, China) to evaluate re-epithelialization. The percentage of re-epithelialization [(distance traversed by epithelium over wound from wound edge/distance between wound edges) $\times 100$ ] was calculated for two sections per wound and was averaged over sections to provide a representative value for each wound ${ }^{22}$. Mason's Trichrome (Solarbio, China) was used to stain sections for evaluation of Collagen deposition. The positive area stained in each image was then quantified by image pro-plus 6.0 (MEDIA CYBERNETICS, Maryland, USA) counting the number of clearly stained pixels above the threshold intensity as previously described ${ }^{18,23}$. Images were captured using a Leica microscope and Leica CellSens Dimension software (Leica Microsystems, Wetzlar, Germany). Slides were scanned at $200 \times$ magnifications.

\section{Immunohistochemical}

Tissue samples of mice were fixed in $10 \%$ buffered formalin ( $0.1 \mathrm{M}$ phosphate buffer, $\mathrm{pH} 7.4$ ) over 24 hours and embedded in paraffin wax (Solarbio, China). All tissues were cut into $3-\mu \mathrm{m}$ sections for staining with an Avidin-Biotin Complex Kit (Vector Laboratories, Burlingame, CA, USA) and a 3,3'-diaminobenzidine kit (Tiangen, Beijing, China). The primary antibody col1 + col3 (ab116223), iNOS (ab15323) and CD163 (ab182422), which are the marker of collagen, M1 and M2 macrophage respectively, were purchased from Abcam, USA. The primary antibody F4/80 (sc-52664), which is a marker of macrophages, was purchased from SANTA, USA. For all staining, inadequate staining samples due to technical problems were excluded. Images were captured using Leica microscope and Leica CellSens Dimension software. Slides were scanned at $200 \times$ magnifications.

\section{Immunoblot analysis}

For the tissue, whole cell extracts were prepared by homogenization in protein extraction solution (10 $\mu$ l of PBS per mg wound tissue, Beyotime, China) containing phenylmethylsulfonyl fluoride (1 mM). Sixty micrograms of protein were charged in each well, separated by $8 \%$ sodium dodecyl sulfatepolyacrylamide gel electrophoresis (SDS-PAGE) and transferred to polyvinylidene difluoride membranes 
(Millipore, USA). The latter were blocked in $5 \%$ bovine serum albumin (BSA) and incubated with a primary antibody overnight at $4{ }^{\circ} \mathrm{C}$ and then incubated with horseradish peroxidase-conjugated secondary antibody for $1 \mathrm{~h}$ at room temperature. Antibodies against iNOS (ab15323) and CD163 (ab182422) were purchased from Abcam, USA. The antibodies of II-1 $\beta$ (sc-32294), TNF-a(C-4) (sc-133192), p65 (sc-56735), p-p65(A-8) (sc-166748), and $\beta$-actin (sc-B0657) were purchased from SANTA CRUZ (USA). Anti-rabbit IgG (\# HAF008), anti-mouse IgG ( \# HAF007) and anti-Rat IgG (\# HAF005) were purchased from R\&D Systems (USA).

\section{Statistical analysis}

Data from the experiments are expressed as means \pm SEM. Data were analyzed with a 1-way ANOVA or Student's t-test by SPSS 19.0 (IBM, Armonk, NY, USA) and Prism 5 (GraphPad Software, La Jolla, CA, USA). Differences between groups of $\mathrm{P} \leq 0.05$ were considered significant.

\section{Results}

\section{Delay wound healing appeared following administration of STZ}

In order to prove the hypothesis, we induced a diabetes model in ICR mice to verify the effect of DE on wound healing and test the mechanism of DE involved in the attenuation of inflammation. Firstly, to confirm that the model of diabetes was successful, the body weight and blood glucose levels (BGLs) of animals were monitored for 2 weeks following the administration of STZ. The results showed that the body weight was decreased at week 2 after injection with STZ (Table 1). And the BGLs started to rise at week 1 after injection with STZ (Fig. 1A). Moreover, it reached to a high level (BGLs $>17 \mathrm{mmol} / \mathrm{L}$ ) at week 2 (Table 2), confirming that the model was successful. We also monitored the BGLs of mice for ten days to verify whether the levels of glucose in blood remained high (Fig. 1A) during the process of experimental. In order to observed whether diabetes could delay wound healing in the model, excisional wounding were administration and topical use at day1. The wound healing process was recorded as described in the methods section. The process of wound healing took 19 days, and the wound areas were then calculated ${ }^{22}$. The percentage of wound healing was measured, and we found that the wound healing was significantly delay in the DM group at days $4,5,6,7,8$, and 9 (Fig. 1B and 1C). These results showed that delay wound healing appeared in diabetic mice and confirmed that the model in this experiment was successfully prepared. 
Table 1

The body weight of mice before and after STZ induced

\begin{tabular}{|c|c|c|}
\hline Treatment & Day 0 (g) & Day 14 (g) \\
\hline Normal & $40.04 \pm 2.02$ & $44.80 \pm 6.46^{* *}$ \\
\hline Diabetes & $40.75 \pm 3.29$ & $34.22 \pm 4.07$ \\
\hline$P$ value & NS & 0.009 \\
\hline \multicolumn{3}{|c|}{$\begin{array}{l}\text { The mice were randomly divided into two groups and detected the bodyweight before and after STZ } \\
\text { induced. Diabetes were induced in the mice with STZ injected intraperitoneally once, at a dose of } \\
100 \mathrm{mg} / \mathrm{kg} \text {. Normal mice were injected with only a saline vehicle. Data are expressed as mean values } \\
\pm \text { SEM. Values represent an average for } 8-16 \text { mice. }{ }^{\star * P}<0.01 \text { by unpaired Student's t-test compared } \\
\text { to normal mice. STZ, streptozotocin. }\end{array}$} \\
\hline
\end{tabular}

Table 2

Effect of STZ treated on blood glucose in ICR mice after 2 weeks.

\begin{tabular}{|c|c|c|c|}
\hline Treatment & Normal & Diabetes & $P$ value \\
\hline Blood glucose & $4.63 \pm 0.83^{\star \star \star}$ & $25.56 \pm 3.86$ & 0.000 \\
\hline
\end{tabular}

\section{Wound healing in DM mice can be improved by DE}

It has been reported that phytochemicals could play a role in wound healing ${ }^{24}$. In order to test whether DE can improve diabetic wound healing, excisional wounding were administration and topical use at day 1. The wound healing process was recorded as described in the methods section. The process of wound healing took 19 days, and the wound areas were then calculated. The percentage of wound healing was measured, and we found that DE treatment could significantly improve the wound healing at days $4,5,6$, 7, 8, and 9 (Fig. 1B and 1C) compare with the model group.

Then, hematoxylin-eosin staining was carried out to observe the effect of DE on the structure of the wounded skin at day 8. Histological assessment showed that the wounds from STZ-induced DM mice had less re-epithelialization on the edge of the wound than the control group, and DE could significantly improve re-epithelialization (Fig. 2A).

Mason's Trichrome stain also showed that DE could increase the level of re-epithelialization of the wounded skin. In addition, wounds treated with DE also showed increased collagen deposition assessed by trichrome staining (Fig. 2B) and IHC staining (Fig. 2C). These results showed that DE could improve late wound healing in DM mice. 
The level of cytokines expression and macrophage accumulation were inhibited by DE in the DM mice wound skin

As we known, the DE could have the ability to attenuate inflammation ${ }^{25}$. Thus, to evaluate the effect of DE on inflammation in DM mice wounds, we measured the release of cytokines from the skin wound tissue. The levels of IL-1 $\beta$ and TNF- $\alpha$ expression were higher in the diabetes group than the normal group and could be significantly decreased by DE (Fig. 3A, 3B and 3C). Macrophages play a critical role in the delayed wound healing process in DM mice ${ }^{15,26}$ by regulated the inflammation responded. To further confirm whether the anti-inflammation effect of $D E$ was related to macrophage, we detected macrophage accumulation by $\mathrm{IHC}$ to evolution the effect of $\mathrm{DE}$ treatment on macrophage accumulation. The results showed that DE could decrease macrophage accumulation significantly compared with the diabetes group (Fig. 3D).

\section{DE could alter the phenotype of macrophage}

Macrophage M1/M2 polarization mediates tissue damage and inflammatory responses ${ }^{27}$ which could play two opposing role during wound healing process ${ }^{13}$. It has a promote inflammation phenotype and also could transformation to a reparative phenotype. In order to confirm which type of macrophages were decreased by DE treatment, pro-inflammatory M1 marker iNOS and anti-inflammatory M2 marker CD163 were detected by immunohistochemistry. The results showed that iNOS was increased and CD163 was decreased in diabetes mice skin. Nevertheless, the iNOS and CD163 expression levels can be recovered by DE (Fig. 4A and 4B). Furthermore, we measured the expression of CD163 from the skin wound tissue by WB to evolution the effect of DE on the alternative of type M2 macrophage. The similar result was observed by immunoblot analysis (Fig. 4C and 4D). These results showed that DE treatment could alter the phenotype of macrophage.

\section{The NF-KB signaling pathway was inhibited by DE in the DM mice wound skin}

The transcription factor NF-KB is a critical regulator of immune and inflammatory responses. And disproportionate increase in activated p65 is integral to the pathogenesis of many chronic diseases ${ }^{28}$. To further understand the molecular mechanisms of the effect of DE on acceleration of diabetes wound healing, canonical NF-KB p65 signaling pathway were detected by immunoblot analysis. The results showed that the level of p65 and p65 phosphorylation were increased in diabetes wounds skin and the activated p65 was attenuated by DE treatment (Fig. 5). These results suggest that DE could attenuate activated canonical NF-kB p65 signaling pathway to decreased inflammatory responses.

\section{Discussion}


Delayed wound healing is one of the most prominent clinical manifestations of diabetes ${ }^{29}$. Further, inflammation plays a critical role in this healing process ${ }^{13}, 30$. With more and more diabetic patient population, the incidence of chronic non-healing wounds is on the rise ${ }^{31}$. Thus, novel therapeutics aimed at controlling inflammation, reducing infection, and improving wound closure is urgently needed. In the present study, we observed that DE could improve wound healing in diabetic mice through decreasing macrophage-mediated inflammation by attenuate activated canonical NF-KB p65 signaling pathway. Our results reveal that $D E$ can inhibit macrophage-mediated inflammation in skin wounds.

Macrophages have been described to have many functions in wounds, including host defense, the promotion and resolution of inflammation, the removal of apoptotic cells, and the support of cell proliferation and tissue restoration following injury. Emerging evidence suggests that macrophage dysfunction is a component of the pathogenesis of non-healing and poorly healing wounds ${ }^{30}$. In addition, extensive research has reported that macrophage accumulation is observed in diabetic patients and plays an important role in wound healing ${ }^{32}$. In type 2 diabetic humans, studies have been found that sustained inflammasome activity in macrophages impairs wound healing and significantly higher number of macrophages was present in the edge of both types of chronic ulcers ${ }^{33}$. Wound macrophages, which derive mostly from circulating monocytes, are recruited to the site of injury and the F4/80 was used to define the recruited macrophages ${ }^{34}$ in the wound area in this study. These researches showed that macrophages promote chronic inflammation in diabetic wounds are established ${ }^{35}$.

Moreover, it has been reported that suppressing NF-KB p65 signaling could decreased the expression of iNOS which is a marker of M1 type macrophage ${ }^{36}$. In addition, previous studies showed that the levels of nuclear p65 and phosphorylated p65 (p-p65) were upregulated in pro-inflammatory phenotype macrophages ${ }^{37,38}$. However, whether activated the $\mathrm{p} 65$ signaling pathway play a role on wound healing in diabetes is still unclear. In this study, we found that the levels of nuclear p65 and p-p65 expression were increased in the skin from STZ-induced DM mice which is frequently used in studies aimed at investigations into diabetes complications ${ }^{19}$. These results showed the potential correlation between macrophage accumulation and p65 signaling pathway in injury skin wound bed. And as expected, our results also showed that the wound healing in DM mice can be improved by DE through inhibiting p65 signaling and macrophage accumulation.

During the proliferative phase, macrophages stimulate proliferation of connective, endothelial and epithelial tissue directly and indirectly. Especially fibroblasts and keratinocytes cells are stimulated by macrophages during this phase to induce and complete extracellular matrix formation and reepithelialization ${ }^{13}$. The abnormal levels of collagen accumulation can disrupt extracellular matrix integrity and lead to the development of chronic wounds ${ }^{39}$. In this paper, we also found that DE could increase re-epithelialization (Fig. $2 \mathrm{~A}$ and $2 \mathrm{~B}$ ) and restore the collagen accumulation in diabetic wounds (Fig. 2C and 2D). 
Inflammatory cytokines increase is observed in wounds of type-1 diabetic patients including CD 40, IL-1a, IL-2, IL-4 and IL-5 ${ }^{40}$. In addition, high levels of the pro-inflammatory molecules IL- $1 \beta$ was expressed in chronic wound macrophages from type 2 diabetic patient ${ }^{20}$. With regard to the high level of macrophage accumulation, we also found that the macrophage-mediated pro-inflammatory cytokines were consequently overexpressed in the wound skin from DM mice and can be ameliorated by DE (Fig. 3). Therefore, DE might accelerate wound healing in diabetes mice through inhibiting the inflammation by blocking NF-KB signal.

\section{Conclusion}

In this study we report that wound healing can be improved by DE through attenuate activated canonical NF-KB p65 signaling pathway in STZ-induced diabetic mice. Our findings provide a novel insight into the therapeutic strategy for diabetic wounds and likely offer $D E$ as a novel potential medicine to treat chronic wounds.

\section{Abbreviations}

Dendrobium officinale Orchid Extract, DE;

STZ, Streptozotocin;

DM, Diabetes mellitus

\section{Declarations}

\section{- Ethics approval and consent to participate}

All mice experiments were performed in the animal facility according to the institutional guidelines and were approved by the Institutional Animal Care and Use Committee of the Yunnan Agricultural University. Adverse events were not observed.

\section{- Consent for publication}

All of authors consent to publication of this study in Journal of Chinese Medicine.

\section{- Availability of data and materials}

The datasets used and/or analyzed during the current study are available from the corresponding author on reasonable request.

\section{- Competing interests}

The authors have no conflict of interest to disclose. 
- Funding

This study was supported by grants from the Yunnan Science and Technology Planning Project (2017ZF003-02). No potential conflicts of interest relevant to this article were reported.

\section{- Authors' contributions}

J.S., X.J.W., and Q.Q.Z. designed the study. Q.Q.Z., C.J.Y, X.Y.Y., J.F.L, M.M.L, and Q.Q.P performed the experiments. Q.Q.Z. and C.J.Y. wrote the manuscript. X.J.W. supervised the overall study. J.S. is the guarantor of this work and, as such, had full access to all the data in the study and takes responsibility for the integrity of the data and the accuracy of the data analysis.

\section{- Acknowledgements}

Not applicable

\section{References}

1. Monteiro-Soares M, Russell D, Boyko EJ, et al. Guidelines on the classification of diabetic foot ulcers (IWGDF 2019). Diabetes/Metabolism Research Reviews. 2020;36(S1):e3273.

2. Organization WH. Global Report on Diabetes 2016. 2019.

3. Kimball AS, Joshi AD, Boniakowski AE, et al. Notch Regulates Macrophage-Mediated Inflammation in Diabetic Wound Healing. Front Immunol. 2017;8:635.

4. Jeffcoate WJ, Harding KG. Diabetic foot ulcers. Lancet. 2003;361(9368):1545.

5. Wu Y, Quan Y, Liu Y, et al. Hyperglycaemia inhibits REG3A expression to exacerbate TLR3-mediated skin inflammation in diabetes. Nat Commun. 2016;7:13393.

6. Jeffcoate WJ, Lipsky BA, Berendt AR, et al. Unresolved issues in the management of ulcers of the foot in diabetes. Diabetic Medicine A Journal of the British Diabetic Association. 2008;25(12):1380.

7. Home C. Self-Reported Visual Impairment Among Persons with Diagnosed Diabetes - United States, 1997-2010. Morbidity Mortality Weekly Report. 2011;60(45):1549-53.

8. Pereira RF, Bártolo PJ. Traditional Therapies for Skin Wound Healing. Adv Wound Care (New Rochelle). 2014;5(5):208-29.

9. Singh S, Young A, McNaught C-E. The physiology of wound healing. Surgery (Oxford). 2017;35(9):473-7.

10. Baltzis D, Eleftheriadou I, Veves A. Pathogenesis and Treatment of Impaired Wound Healing in Diabetes Mellitus: New Insights. Advances in Therapy. 2014;31(8):817-36.

11. Yuan Y, Das Sushant K, Li M. Vitamin D ameliorates impaired wound healing in streptozotocininduced diabetic mice by suppressing NF-KB-mediated inflammatory genes. Bioscience Reports. 2018;38(2). 
12. Pauline Bannon SW, Restivo T, Campbell L, Hardman MJ. KAM. Diabetes induces stable intrinsic changes to myeloid cells that contribute to chronic inflammation during wound healing. Disease Models \& Mechanisms. 2013: 1-40.

13. Koh TJ, DiPietro LA. Inflammation and wound healing: the role of the macrophage. Expert Rev Mol Med. 2011;13:e23.

14. Mahdavian Delavary B, van der Veer WM, van Egmond M, Niessen FB, Beelen RH. Macrophages in skin injury and repair. Immunobiology. 2011;216(7):753-62.

15. Boniakowski AE, Kimball AS, Jacobs BN, Kunkel SL, Gallagher KA. Macrophage-Mediated Inflammation in Normal and Diabetic Wound Healing. The Journal of Immunology. 2017;199(1):1724.

16. Ng TB, Liu J, Wong JH, et al. Review of research on Dendrobium, a prized folk medicine. Appl Microbiol Biotechnol. 2012;93(5):1795-803.

17. Pil YS, Young Hee M, Ran H, et al. Protective effects of epigallocatechin gallate (EGCG) on streptozotocin-induced diabetic nephropathy in mice. Acta Histochemica. 2014;116(8):1210-5.

18. Huang $Y-W$, Zhu Q-Q, Yang $X-Y$, et al. Wound healing can be improved by (-)-epigallocatechin gallate through targeting Notch in streptozotocin-induced diabetic mice. The FASEB Journal. 2018;0(0): fj.201800337R.

19. Dekel Y, Glucksam Y, Elron-Gross I, Margalit R. Insights into modeling streptozotocin-induced diabetes in ICR mice. Lab Anim (NY). 2009;38(2):55-60.

20. Rita E, Mirza MM, Fang, William J. Ennis aTJK. Blocking Interleukin-1b Induces a Healing-Associated Wound Macrophage Phenotype and Improves Healing in Type 2 Diabetes. Diabetes. 2013;62:257987.

21. Gallagher KA, Joshi A, Carson WF, et al. Epigenetic changes in bone marrow progenitor cells influence the inflammatory phenotype and alter wound healing in type 2 diabetes. Diabetes. 2015;64(4):142030 .

22. Huang Y-W, Zhu Q-Q, Yang X-Y, et al. Wound healing can be improved by (-)-epigallocatechin gallate through targeting Notch in streptozotocin-induced diabetic mice. The FASEB Journal. 2018;33(1):953-64.

23. Chen J, Li Y, Zhu Q, et al. Anti-skin-aging effect of epigallocatechin gallate by regulating epidermal growth factor receptor pathway on aging mouse model induced by d -Galactose. Mechanisms of Ageing Development. 2017;164:1-7.

24. Shah A, Amini-Nik S. The Role of Phytochemicals in the Inflammatory Phase of Wound Healing. Int J Mol Sci. 2017;18(5):1068.

25. Yang J, Chen H, Nie Q, Huang X, Nie S. Dendrobium officinale polysaccharide ameliorates the liver metabolism disorders of type II diabetic rats. Int J Biol Macromol. 2020.

26. Kim SY, Nair MG. Macrophages in wound healing: activation and plasticity. Immunology Cell Biology. 2019;97(3):258-67. 
27. Tian L, Li W, Yang L, et al. Cannabinoid Receptor 1 Participates in Liver Inflammation by Promoting M1 Macrophage Polarization via RhoA/NF-kappaB p65 and ERK1/2 Pathways, Respectively, in Mouse Liver Fibrogenesis. Front Immunol. 2017;8:1214.

28. Giridharan S, Srinivasan M. Mechanisms of NF-KB p65 and strategies for therapeutic manipulation. J Inflamm Res. 2018;11:407-19.

29. Landén NX, Li D, Ståhle M. Transition from inflammation to proliferation: a critical step during wound healing. Cellular Molecular Life Sciences. 2016;73(20):3861-85.

30. Hatanaka E, Monteagudo PT, Marrocos MS, Campa A. Neutrophils and monocytes as potentially important sources of proinflammatory cytokines in diabetes. Clinical Experimental Immunology. 2006;146(3):443-7.

31. Lontchiyimagou E, Sobngwi E, Matsha TE, Kengne AP. Diabetes Mellitus and Inflammation. Current Diabetes Reports. 2013;13(3):435-44.

32. Sen CK, Gordillo GM, Roy S, et al. Human Skin Wounds: A Major and Snowballing Threat to Public Health and the Economy. Wound Repair Regeneration. 2009;17(6):763.

33. Rita E. Mirza MMF, Eileen M. Weinheimer-Haus WJ, Ennis, Timothy J. Koh. Sustained Inflammasome Activity in Macrophages Impairs Wound Healing in Type 2 Diabetic Humans and Mice. Diabetes. 2014;63:1103-14.

34. Brancato SK, Albina JE. Wound Macrophages as Key Regulators of Repair: Origin, Phenotype, and Function. The American Journal of Pathology. 2011;178(1):19-25.

35. Trengove NJ, PhD HB-OD, FRACS MCSD. Mitogenic activity and cytokine levels in non-healing and healing chronic leg ulcers. Wound Repair Regeneration. 2010;8(1):13-25.

36. He Y, Ma X, Li D, Hao J. Thiamet G mediates neuroprotection in experimental stroke by modulating microglia/macrophage polarization and inhibiting NF-KB p65 signaling. Journal of Cerebral Blood Flow Metabolism. 2016;37(8):2938-51.

37. Delprat V, Tellier C, Demazy C, Raes M, Feron O, Michiels C. Cycling hypoxia promotes a proinflammatory phenotype in macrophages via JNK/p65 signaling pathway. Scientific Reports. 2020;10(1):882.

38. Wang J, Cao Y, Liu Y, et al. PIM1 inhibitor SMI-4a attenuated lipopolysaccharide-induced acute lung injury through suppressing macrophage inflammatory responses via modulating p65 phosphorylation. Int Immunopharmacol. 2019;73:568-74.

39. Lucas $T$, Waisman A, Ranjan R, et al. Differential roles of macrophages in diverse phases of skin repair. J Immunol. 2010;184(7):3964-77.

40. Chatzigeorgiou A, Harokopos V, Mylona-Karagianni C, Tsouvalas E, Aidinis V, Kamper EF. The pattern of inflammatory/anti-inflammatory cytokines and chemokines in type 1 diabetic patients over time. Annals of Medicine. 2010;42(6):426.

\section{Figures}




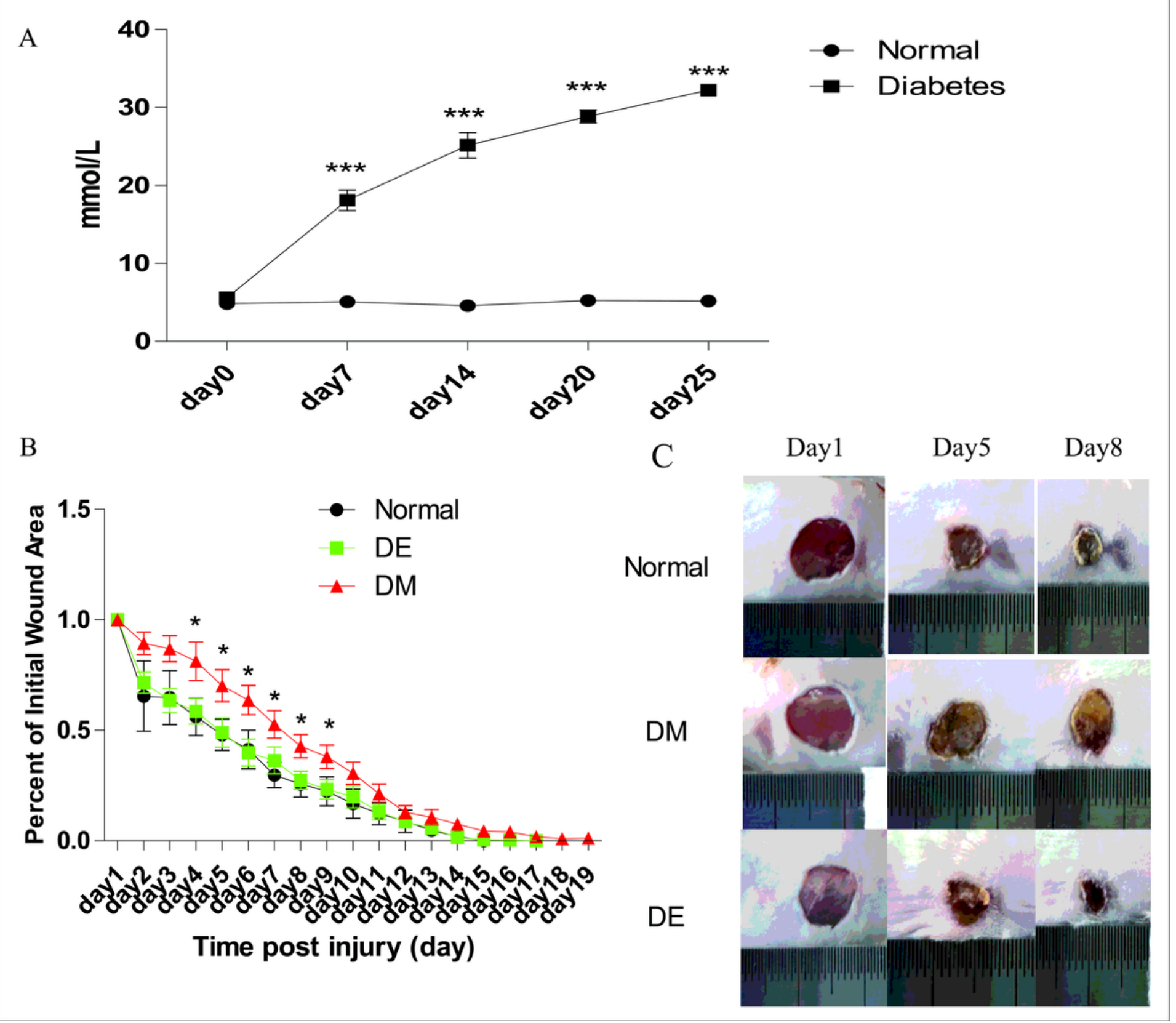

\section{Figure 1}

Delay wound healing appeared following administration of STZ. STZ was freshly dissolved in cold citrate buffer and a single i.p. injection of $100 \mathrm{mg}$ per kg body weight was administered at day 0 , and BGLs were measured at day 0 , day 7 , day 14 , day20, and day 25 after STZ injection (A). Values represent an average for 8-16 mice. Error bars represent s.e.m. From week 1 onward, the differences between normal and model groups were significant $(P<0.001)$. The curves are non-theoretical, drawn to emphasize trends in the data. Wounds were created using 8-mm punch biopsies on the backs of STZ-induced DM mice and normal mice. Change in wound area was recorded daily using a digital camera and Image $J(N I H)$ until complete healing was observed (B \& C). Data are pooled from two experiments $(n=6$ wounds in 6 mice/group, repeated 2x). BGLs, blood glucose levels. STZ, streptozotocin. 


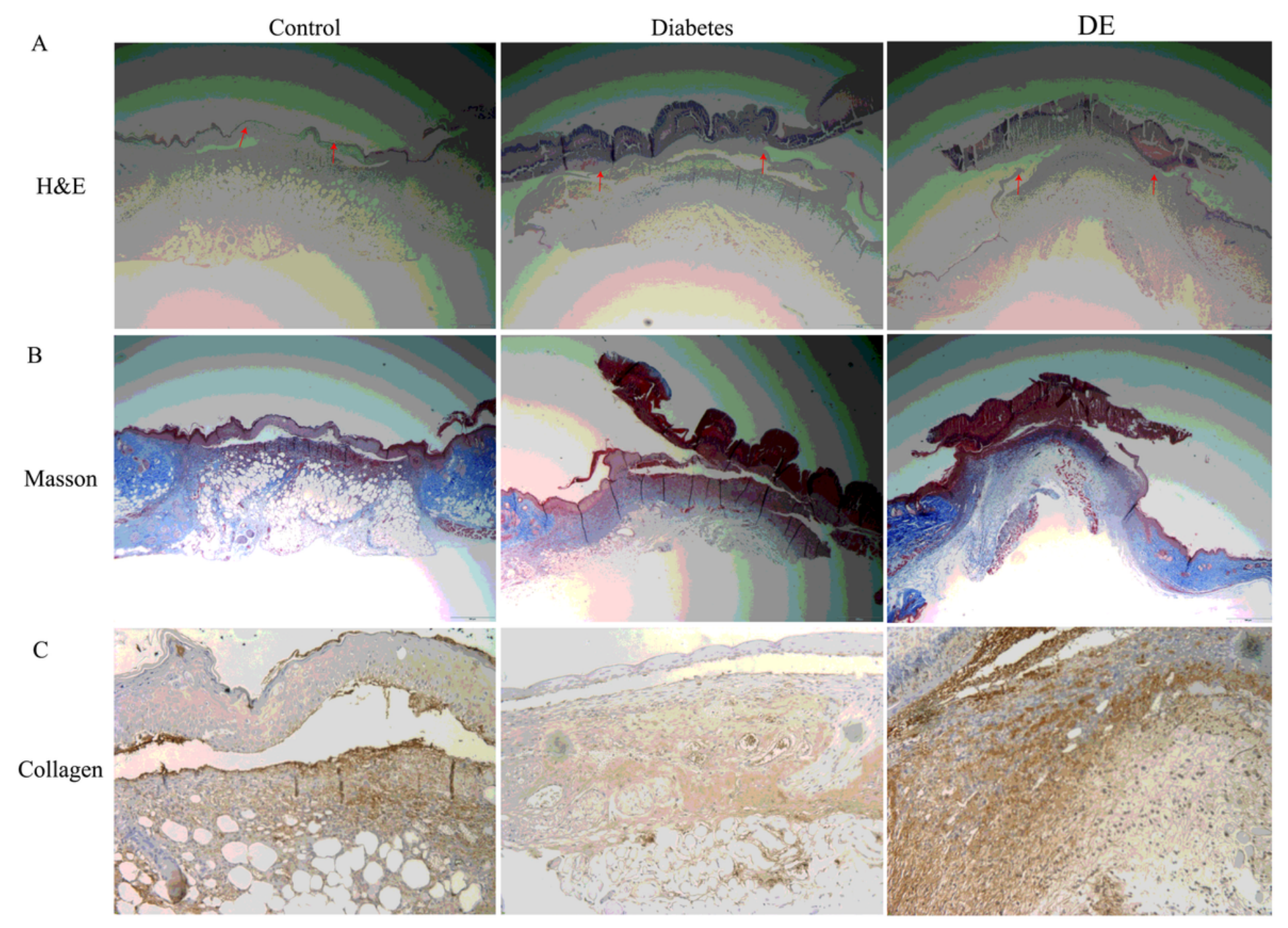

\section{Figure 2}

Wound healing in DM mice can be improved by DE. Histopathological images showing paraffin sections stained with $\mathrm{H} \& \mathrm{E}(\mathrm{A})$ and collagen deposition (B) of wounds on post-injury day 8 . The diabetes group shows less re-epithelialization (arrows indicate ends of migrating epithelial) and collagen deposition (blue area) compared with both the normal and DE-treated groups. For IHC detection, 3- $\mu \mathrm{m}$ sections were stained for collagen detection (C).Data are pooled from two experiments ( $n=6$ wounds in 6 mice/group, repeated $2 \times$ ). Statistical analysis was performed using one-way ANOVA. All data are expressed as mean \pm SEM. DM, diabetes mellitus. DE, Dendrobium officinale Orchid Extract. 


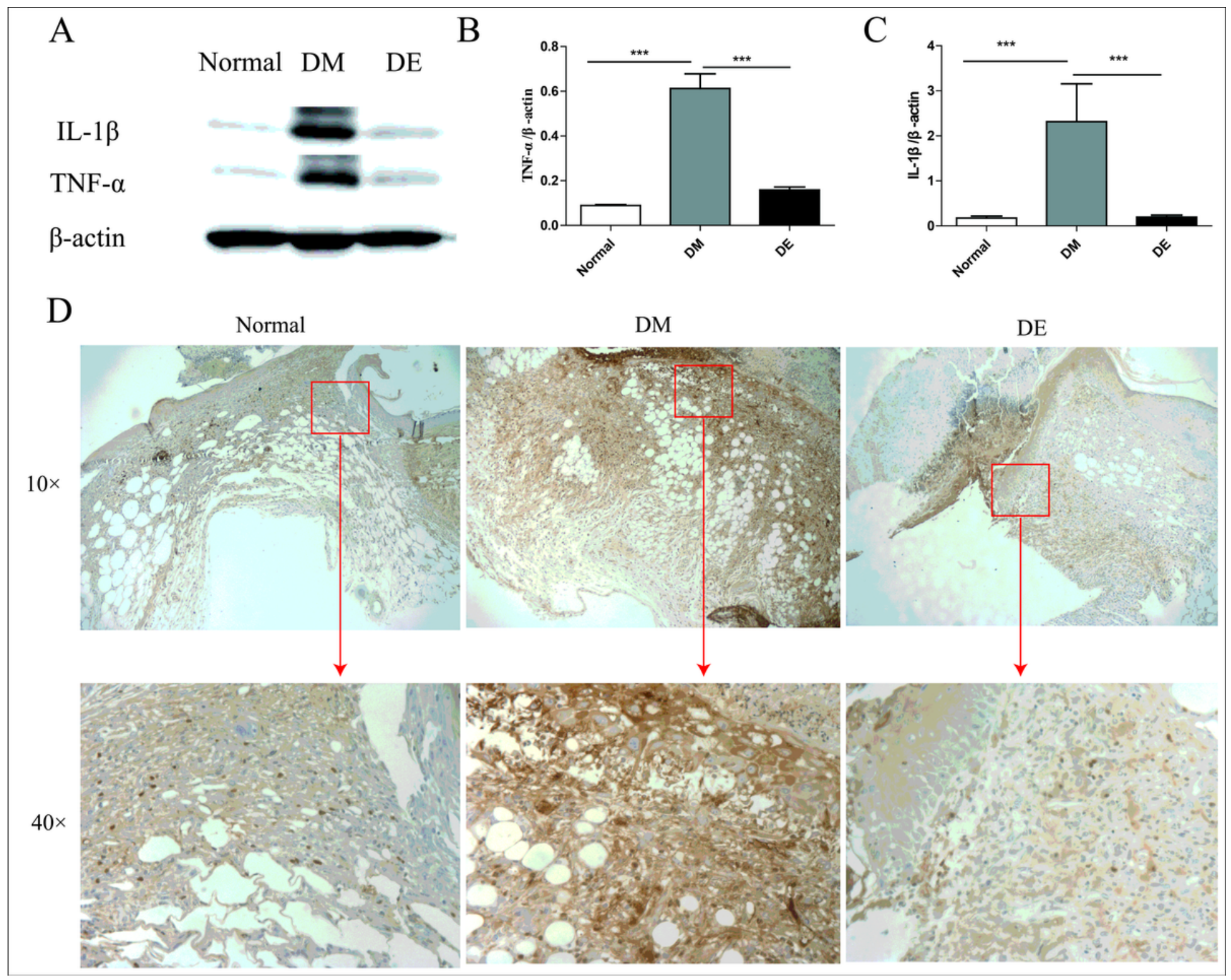

\section{Figure 3}

The level of cytokines expression and macrophage accumulation were inhibited by DE in the DM mice wound skin. Wounds in DM mice were treated with DE or placebo and harvested at day 8 post-injury. Tissue was obtained from mice by performing punch biopsies on the backs of animals. And then, the tissue was homogenized in protein extraction solution and $60 \mu \mathrm{g}$ for each well for detected. To measure the protein expressions of pro-inflammatory marker IL-1 $\beta$ and TNF- $\alpha(A \& B)$, tissues were treated as described earlier using western blotting. The tissue was obtained from the backs of mice was homogenized in protein extraction solution and $60 \mu \mathrm{g}$ for each well for detected. Immunohistochemisty was performed on paraffin sections stained for $F 4 / 80$ (C). Brown area (red box shown) indicated positive $F 4 / 80$ staining. For all graphs, bars $=$ mean $\pm S E M, n=5$. Statistical analysis was performed using oneway ANOVA. *** Mean value was significantly different for the EGCG and normal group at the same time point compared with the diabetes group. DM, diabetes mellitus. DE, Dendrobium officinale Orchid Extract. 


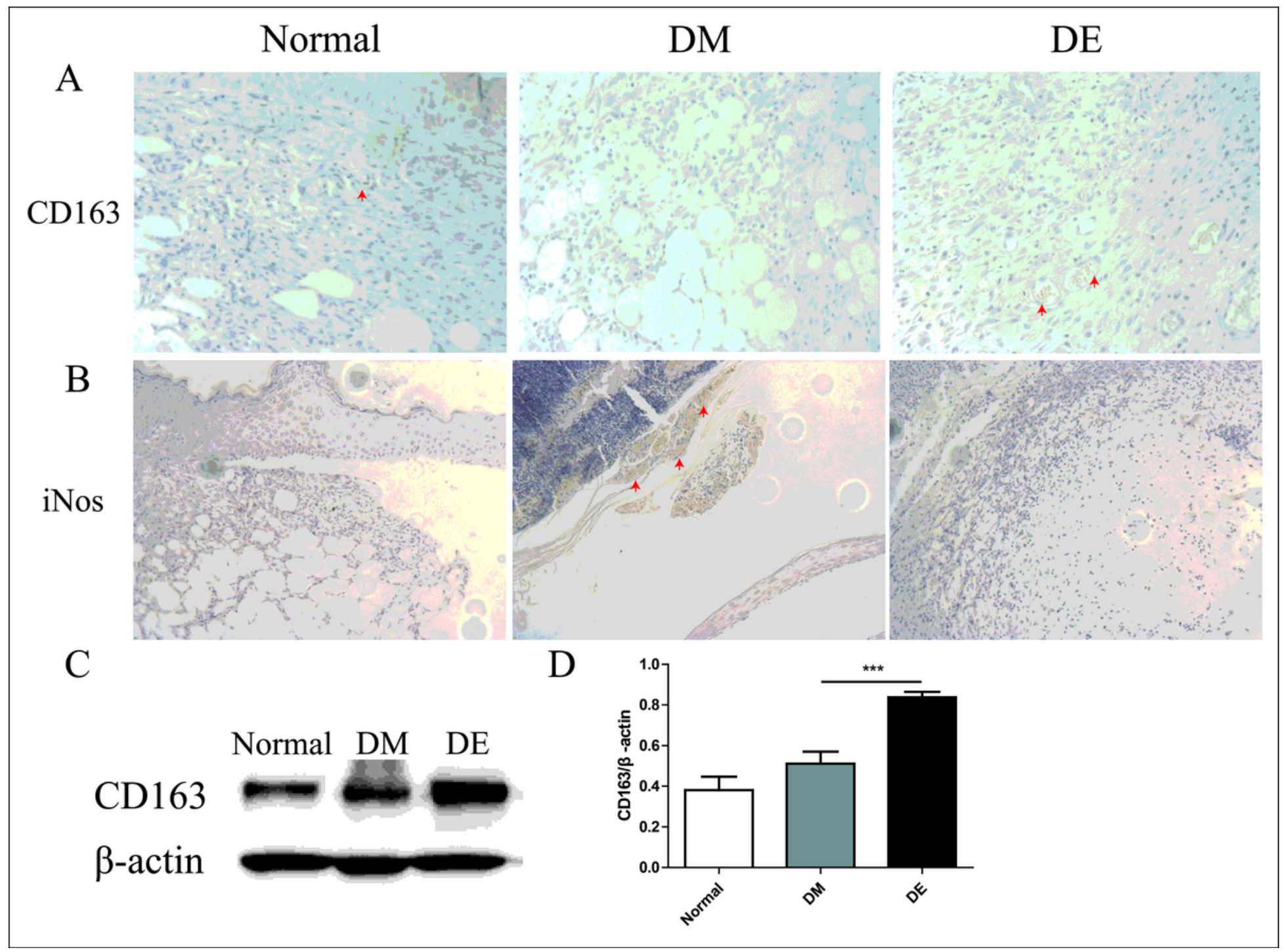

\section{Figure 4}

DE could alter the phenotype of macrophage. Wounds in DM mice were treated with DE or placebo and harvested at day 8 post-injury. Tissue was obtained from mice by performing punch biopsies on the backs of animals. Immunohistochemisty was performed on paraffin sections stained for M1 and M2 phenotype macrophage (A \& B). The brown cell as positive area is indicate by arrows, iNOS (black arrows) and CD163 (green arrows). The tissues from the backs of DM mice were homogenized in protein extraction solution and $60 \mu \mathrm{g}$ for each well for detected. The expression of CD163(C) was measured by western blotting. For all graphs, bars $=$ mean $\pm S E M, n=5$. Statistical analysis was performed using oneway ANOVA. ${ }^{\star \star \star}$ Mean value was significantly different for the EGCG and normal group at the same time point compared with the diabetes group. DM, diabetes mellitus. DE, Dendrobium officinale Orchid Extract. 


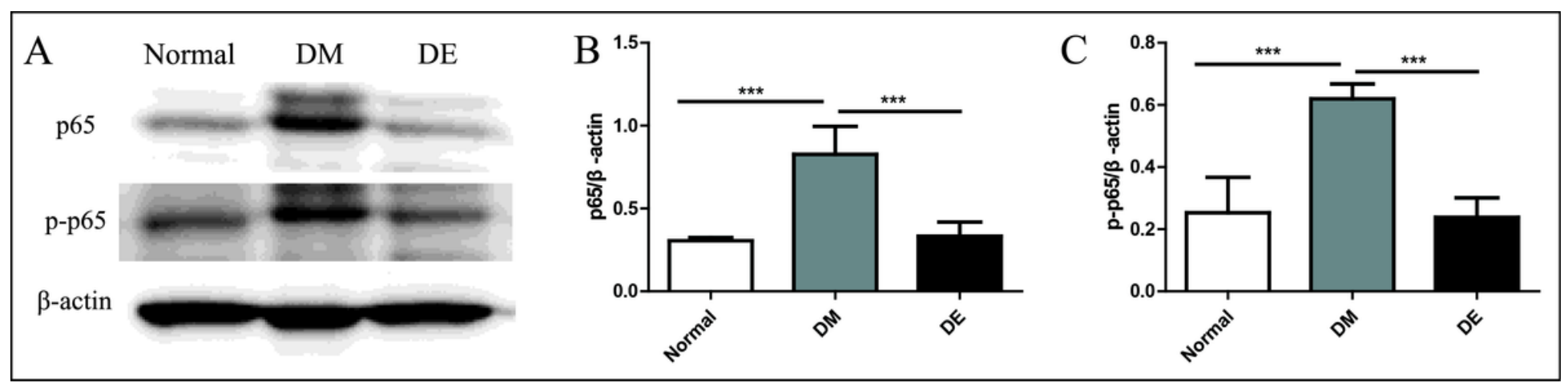

\section{Figure 5}

The NF-KB signaling pathway was inhibited by DE in the DM mice wound skin. Tissue was obtained from mice by performing punch biopsies on the backs of animals. And then, the tissue was homogenized in protein extraction solution and $60 \mu \mathrm{g}$ for each well for detected. To demonstrate the effect of DE on NFKB signaling pathway, p65 and p-p65 were detected by western blotting $(A)$, and the OD values (B) were calculated using the Image $\mathrm{J}$ software $(\mathrm{NIH})$. For all graphs, bars $=$ mean $\pm \mathrm{SEM}$ and $\mathrm{n}=3$ for both experiments, repeated $3 \times$. ${ }^{* \star}$ Mean value significantly different from that for $D M$ group, $P<0.001$. DM, diabetes mellitus. DE, Dendrobium officinale Orchid Extract. 